\title{
Formes frustes des fentes labio-palatines: présentation d'un cas clinique
}

\author{
Manon Devisse ${ }^{1}$, Caroline Darbin ${ }^{1}$, Julie Lelièvre ${ }^{1}$, Cédric Lansonneur ${ }^{1}$, \\ Yves Gauvin ${ }^{1}$, Sylvie Boisramé-Gastrin ${ }^{1}$
}

${ }^{1}$ Service d'Odontologie, CHU, 29200 Brest, France

${ }^{2}$ Service d'Odontologie, UFR d'Odontologie, 29238 Brest Cedex 3, France

manon.devisse@chu-brest.fr

Les fentes labio-palatines sont les dysmorphoses buccales congénitales les plus fréquentes (1/600 naissances). Elles peuvent être associées à des syndromes polymalformatifs. Malgré une étiologie inconnue, l'hérédité serait un facteur prédominant et il existerait des facteurs prédisposants comme la toxicomanie maternelle, l'alcoolisme parental, la prise de certains médicaments pendant la grossesse (Cooney).

Elles résultent d'une absence ou d'une insuffisance de fusion des différents bourgeons faciaux se déroulant de la 5ème et la 7ème semaine intra-utérine, aboutissant à des fentes plus ou moins marquées : les fentes labiales, labio-alvéolaires, palato-vélaires, unilatérales ou bilatérales, complètes ou incomplètes (Yuzuriha et al. 2008).

Les fentes labio-palatines de forme fruste, également appelées mineures ou abortives, n'ont pas d'incidence sur l'esthétique : moins visibles, elles sont moins connues. Elles se caractérisent par une luette bifide et elles ont également des conséquences dentaires comme par exemple des dents gémellées. Les anomalies dentaires peuvent affecter la denture temporaire et la denture définitive. La dent la plus concernée par les anomalies étant l'incisive latérale bordant la fente ; cela induit une variation du nombre de dents (dédoublement de l'incisive latérale souvent déplacée vers le palais on agénésie) ou de leur forme.

Dans les formes mineures, il existe également la fente palatine sous-muqueuse (FPSM) qui est une sous-catégorie de fente palatine pour laquelle la plupart des individus atteints ne ressentent aucun symptôme. Elle touche le palais mou et peut entrainer une insuffisance vélo-pharyngée qui affecte la qualité et l'intelligibilité de l'élocution (Nasser et al. 2008).

Ces fentes frustes nécessitent ainsi une prise en charge transdisciplinaire (ORL, chirurgien-dentiste, orthodontiste et orthophoniste). Elles ne doivent pas rester méconnues du chirurgien-dentiste afin de permettre une prise en charge adaptée (Vogels et al. 2011)

Un enfant âgé de 9 ans est adressé dans le service d'Odontologie du Centre hospitalier régional universitaire de Brest pour la persistance unilatérale de l'incisive latérale temporaire maxillaire droite. A l'examen clinique, on retrouvait une duplication de cette dent (52 et 52 bis) et on observait une bifidité de la luette. L'orthopantomogramme a objectivé la rétention de l'incisive latérale permanente elle-même gémellée (12 et 12 bis) et de la canine permanente. 52 bis et 12 bis, situées en position mésiale par rapport aux canines temporaire et permanente ont été extraites ; ceci a ouvert un chemin d'éruption normal de la canine permanente ectopique.

Le chirurgien-dentiste a un rôle dans la prise en charge de ces patients d'une part pour établir un diagnostic précoce et d'autre part pour réaliser un traitement approprié permettant d'éviter des malpositions, des interférences occlusales et des rétentions pouvant être à l'origine de complications infectieuses. Et il reste en étroite collaboration avec l'orthodontiste si nécessaire. 